\title{
TYCHONOFF CUBES ARE COSET SPACES
}

\section{CARL EBERHART}

1. Introduction. Let $T$ denote a Tychonoff cube (some infinite product of unit intervals) and let $Q$ denote the Hilbert cube (the countably infinite product of intervals). It has been known for some time that $Q$ is a homogeneous space. Consequently $T$ is also homogeneous, since it can be written as a product of Hilbert cubes. In this note we shall show that $T$ is a coset space.

2. Preliminaries. The question of when a homogeneous space $X$ is a coset space has been considered by Ford [1] and Mostert [4], among others. Ford showed that it is necessary that $X$ be completely regular and Hausdorff, and he proposed a very interesting sufficient (but not necessary) condition. Call an open set $U$ in $X$ an SLH set provided that for each $x, y \in U$ there is a $g \in G(X)$, the group of homeomorphisms of $X$, such that $g(x)=y$ and $g(z)=z$ for all $z \in X \backslash U$. The space $X$ is called a strongly locally homogeneous (SLH) space if the SLH sets form a basis for the topology on $X$. Mostert stated the correct theorem using the SLH property.

THEOREM 1. Let $X$ be a completely regular Hausdorff space which is homogeneous and SLH. Then the topology on $G(X)$ induced by the uniformity on $X$ obtained from the Stone-Cech compactification of $X$ is reasonable in the sense that $G(X)$ is a topological group and the natural map $\mu: G(X) / G(X)_{x} \rightarrow X$ is a homeomorphism, where $G(X)_{x}$ denotes the stable group at some $x \in X$.

We shall make use of the following observation.

Lemma 2. If $X$ is homogeneous and the SLH sets in $X$ contain a basis for the topology on $X$ at some point $x \in X$, then $X$ is an SLH space.

Proof. Let $U$ be an open set containing $y \in X$. Let $h \in G(X)$ such that $h(y)=x$. Let $V$ be an SLH set containing $x$ such that $V \subset h(U)$. Clearly $h^{-1}(V)$ is an SLH set containing $y$ and lying in $U$.

3. $T$ is an SLH space. A property of $G(Q)$ discovered by $\mathrm{R}$. Wong [5] provides the key to our argument. A mapping $H: I \times Q \rightarrow Q$ is called an isotopy if $H_{t}=H \mid t \times Q$ is in $G(Q)$ for all $t \in I$. When $H$ is an isotopy, then $H_{0}$ and $H_{1}$ are said to be isotopic.

Received by the editors November 19, 1966. 
THEOREM 3 (WoNG). Every element of $G(Q)$ is isotopic to the identity of $G(Q)$.

Theorem 4. Tis an SLH space.

Proof. Let $T=\prod_{\beta \in \Lambda} I_{\beta}$ and let $B$ consist of the collection of open sets of the form

$$
\prod_{\beta \in F}\left(\frac{1}{2}-\frac{1}{2}^{n}, \frac{1}{2}+\frac{1}{2}^{n}\right) \times \prod_{\beta \in \Lambda \backslash F} I_{\beta}
$$

where $F$ is a finite subset of $\Lambda$ and $n$ is a natural number. Then $B$ forms a basis for $T$ at the point $m$, each of whose coordinates is $\frac{1}{2}$. We will show that each member of $B$ is an SLH set. The theorem then follows from Lemma 2.

Choose

$$
B=\prod_{\beta \in F} I_{\beta n} \times \prod_{\beta \in \Lambda \backslash F} I_{\beta} \in \mathbb{B}
$$

where

$$
F=\{1,2, \cdots, r\} \subset \Lambda \text { and } I_{\beta n}=\left(\frac{1}{2}-\frac{1}{2}^{n}, \frac{1}{2}+\frac{1}{2}^{n}\right) \subset I_{\beta} .
$$

Then we may write

and

$$
T=I^{r-1} \times I_{r} \times \prod_{\beta \in \Omega} Q_{\beta}
$$

$$
B=U^{r-1} \times I_{r n} \times \prod_{\beta \in \Omega} Q_{\beta}
$$

where

$$
I^{r-1}=\prod_{\beta \in F \backslash r} I_{\beta}, \quad U^{r-1}=\prod_{\beta \in F \backslash r} I_{\beta n}, \quad \text { and } \quad \prod_{\beta \in \Omega} Q_{\beta}=\prod_{\beta \in \Delta \backslash F} I_{\beta} .
$$

Choose $x \in B$. We wish to construct a homeomorphism $h \in G(T)$ such that $h(x)=m$ and $h(z)=z$ for all $z \in T \backslash B$. This is done in several steps. For $A \subset \Omega$,

$$
p_{A}: \prod_{\beta \in \Omega} Q_{\beta} \rightarrow \prod_{\beta \in \boldsymbol{A}} Q_{\beta}
$$

denotes the natural projection.

1. Let $f \in G\left(I^{r-1}\right)$ such that 


$$
f p_{F \backslash r}(x)=p_{F \backslash r}(m) \quad \text { and } \quad f(z)=z
$$

for all $z \in I^{r-1} \backslash U^{r-1}$. This is possible since $U^{r-1}$ is an open $r-1$ cell contained in the interior of $I^{r-1}$ and $p_{F \backslash r}(x), p_{F \backslash r}(m)$ are in $U^{r-1}$.

2. Let $g \in G\left(I_{r}\right)$ such that $g p_{r}(x)=g p_{r}(m)$ and $g(z)=z$ for all $z \in I_{r} \backslash I_{r n}$.

3. For each $\beta \in \Omega$, let $g_{\beta} \in G\left(Q_{\beta}\right)$ such that $g_{\beta} p_{\beta}(x)=p_{\beta}(m)$. The homogeneity of each $Q_{\beta}$ assures the existence of $g_{\beta}$.

4. For each $\beta \in \Omega$, use Theorem 3 to construct an isotopy $H^{\beta}: I_{r} \times Q_{\beta}$ $\rightarrow Q_{\beta}$ so that $H_{t}^{\beta}=\mathrm{id} Q_{\beta}$, the identity on $Q_{\beta}$, for $t \in I_{r} \backslash I_{r n}$ and $H_{1 / 2}^{\beta}=g_{\beta}$.

5. Define

$$
k: I_{r} \times \prod_{\beta \in \Omega} Q_{\beta}=I_{r} \times \prod_{\beta \in \Omega} Q_{\beta}
$$

by

$$
k(t \times y)=t \times \prod_{\beta \in \Omega} H_{t p_{\beta}}^{\beta}(y) .
$$

Note that $k \in G\left(I_{r} \times \prod_{\beta \in \Omega} Q_{\beta}\right)$ and $k(z)=z$ for

$$
z \in\left(I_{r} \times \prod_{\beta \in \Omega} Q_{\beta}\right) \backslash\left(I_{r n} \times \prod_{\beta \in \Omega} Q_{\beta}\right) .
$$

6. Now let $h \in G(T)$ be defined by $h=(f \times k) \circ g^{*}$ where $g^{*}$ denotes the element of $G(T)$ given by $p_{\beta} g^{*}(z)=p_{\beta}(z)$ for $\beta \neq r$ and $p_{r} g^{*}(z)=g p_{r}(z)$. One may check that $h$ is the required homeomorphism.

Since every point of $B$ can be moved to $m$ by a homeomorphism leaving $T \backslash B$ point wise fixed, it follows that $B$ is an SLH set. This completes the proof.

The following is an immediate corollary of Theorem 1 and Theorem 4 .

Corollary 5. $T$ is a coset space.

REMARK 6. It is interesting that although $T$ is a coset space, it is not a coset space of any compact group. This follows from a theorem of Borel which states that an acyclic coset space of a compact group is a point [2, p. 310].

A theorem due to Madison [3] states that any continuous associative multiplication with identity on a continuum which admits a group structure is itself a group multiplication. It can easily be seen that $T$ admits a continuous associative multiplication with identity which is not a group multiplication. Hence we have an example to 
show that "group structure" can not be weakened to "coset space" in Madison's theorem.

\section{REFERENCES}

1. L. R. Ford, Jr., Homeomorphism groups and coset spaces, Trans. Amer. Math. Soc. 77 (1954) 490-497.

2. K. H. Hofmann and Paul S. Mostert, Elements of compact semigroups, Merrill, Columbus, Ohio, 1966.

3. Bernard Madison, Clans on coset spaces, Doctoral dissertation, University of Kentucky, Lexington, 1966.

4. Paul S. Mostert, Reasonable topologies for homeomorphism groups, Proc. Amer. Math. Soc. 12 (1961), 598-602.

5. Raymond Yen-Tin Wong, On homeomorphisms of infinite dimensional product spaces, Doctoral dissertation, Louisiana State University, Baton Rouge, 1966.

UNIVERSITY OF KENTUCKY 